\title{
Atomic nonclassicality quasiprobabilities
}

\author{
T. Kiesel and W. Vogel \\ Arbeitsgruppe Quantenoptik, Institut für Physik, Universität Rostock, D-18051 Rostock, Germany \\ S. L. Christensen, J.-B. Béguin, J. Appel, E. S. Polzik \\ QUANTOP, Niels Bohr Institute, University of Copenhagen, Blegdamsvej 17, 2100 København Ø, Denmark
}

\begin{abstract}
Although nonclassical quantum states are important both conceptually and as a resource for quantum technology, it is often difficult to test whether a given quantum system displays nonclassicality. A simple method to certify nonclassicality is introduced, based on easily accessible collective atomic quadrature measurements, without the need of full state tomography. The statistics is analyzed beyond the ground-state noise level, by direct sampling of a regularized atomic quadrature quasiprobability. Nonclassicality of a squeezed ensemble of $2 \cdot 10^{5}$ Cesium atoms is demonstrated, with a significance of up to 23 standard deviations.
\end{abstract}

PACS numbers: 03.65.Ta, 75.10.Jm, 42.50.Lc, 42.50.Dv

\section{INTRODUCTION}

The difference between the classical and quantum mechanical description of physical systems is often discussed in terms of nonclassicality. This topic is of general interest, since it gives insight into the reasons why a classical picture of nature has to fail, and provides methods to explore what a quantum mechanical system can do which a classical system cannot. In particular, such issues are of great importance for numerous fields in quantum optics, quantum computation, quantum information, and related research areas.

Nonclassicality of the harmonic oscillator has been well understood during the last years. Its definition is based on the coherent states $|\alpha\rangle$, parameterized by a complex amplitude $\alpha$. The evolution of these pure quantum states most closely corresponds to the classical evolution of position and momentum. They are minimum uncertainty states with a stable Gaussian wave packet. In this sense they represent the closest analogue to the classical behavior. Furthermore, they allow to decompose any quantum state in the form

$$
\hat{\rho}=\int d^{2} \alpha P(\alpha)|\alpha\rangle\langle\alpha|,
$$

where $P(\alpha)$ is the Glauber-Sudarshan $P$ function [1, 2]. In this representation, an arbitrary quantum state formally resembles a statistical mixture of (classical) coherent states. However, for many states $P(\alpha)$ may show negativities, which cannot be interpreted in terms of statistical probability densities. Such states are consequently referred to as nonclassical [3].

Atomic coherent states (ACS) have already been developed in 4. It has been shown that their interaction with a classical electric and magnetic field can be understood classically, cf. [5], and any density operator can be formally written as a statistical average over ACS. This remarkable property allows one to define nonclassicality in a way closely corresponding to that of the harmonic oscillator [6, 7]. Specific nonclassical properties of atomic systems have already been discussed in previous publications, such as spin squeezing [8] 10] and squeezing in Ramsey spectroscopy [11. The creation of entanglement in spin systems [12, 13] and the relation between entanglement and squeezing 14 have also been studied. Recently, nonclassicality has been examined in terms of measurable probability distributions [15].

The present manuscript addresses a method to visualize nonclassicality of atomic systems, characterized by angular momentum states with a large orientation in the $z$-direction and small fluctuations along the two orthogonal directions. To provide a simple and experimentally accessible condition for the detection of the nonclassical features of the system, we introduce the concept of a regularized atomic quadrature quasiprobability (AQQP). Our method is applied to experimental data from approximately $10^{5}$ two-level atoms, which were collectively prepared in an atomic spin-squeezed state. It uncovers the nonclassical properties, even for weak squeezing.

The paper is organized as follows: In Sec. II, we give a short introduction into the quantum mechanical description of the angular momentum, and review the HolsteinPrimakoff approximation. Then, we explain the verification of nonclassical effects by nonclassicality quasiprobabilities and define nonclassicality quadrature distributions. The experimental results, including the setup and data analysis, are given in Sec. III. Finally, we summarize our conclusions in Sec. IV.

\section{ATOMIC COHERENT STATES}

\section{A. Basic relations}

Formally, the atomic ensemble under study can be characterized as an angular momentum system. In quantum mechanics, the angular momentum is described by a vector valued operator $\hat{\vec{J}}=\left(\hat{J}_{x}, \hat{J}_{y}, \hat{J}_{z}\right)$, satisfying the 
commutation relations (setting $\hbar=1$ ):

$$
\left[\hat{J}_{x}, \hat{J}_{y}\right]=\mathrm{i} \hat{J}_{z}, \quad\left[\hat{J}_{y}, \hat{J}_{z}\right]=\mathrm{i} \hat{J}_{x}, \quad\left[\hat{J}_{z}, \hat{J}_{x}\right]=\mathrm{i} \hat{J}_{y}
$$

Furthermore, we introduce the angular momentum ladder operators $\hat{J}_{ \pm}=\hat{J}_{x} \pm \mathrm{i} \hat{J}_{y}$, whose commutator is given by $\left[\hat{J}_{+}, \hat{J}_{-}\right]=2 \hat{J}_{z}$. Throughout this work, we keep the quantum number $j$ fixed, while the quantum number $m$ takes values in $\{-j,-j+1, \ldots, j\}$.

The foundation for the discussion of nonclassicality is typically given by the coherent states, being defined as 16

$$
|\theta, \varphi\rangle=\exp \left\{\left(\theta e^{\mathrm{i} \varphi} \hat{J}_{-}-\theta e^{-\mathrm{i} \varphi} \hat{J}_{+}\right) / 2\right\}|j ; j\rangle,
$$

where the fiducial state $|j ; j\rangle$ is the common eigenstate of the operators $\hat{J}^{2}$ and $\hat{J}_{z}$ with maximum eigenvalue for the latter operator. These coherent states can be seen as the analog to a classical angular momentum state. They have minimum uncertainty in $\hat{J}^{2}$, and their expectation value of the projection of $\hat{\vec{J}}$,

$\vec{n}(\theta, \varphi) \cdot \hat{\vec{J}}=\sin (\theta) \cos (\varphi) \hat{J}_{x}+\sin (\theta) \sin (\varphi) \hat{J}_{y}+\cos (\theta) \hat{J}_{z}$

is exactly equal to $j$, without uncertainty, such that the state $|\theta, \varphi\rangle$ can be understood as pointing exactly in the direction of $\vec{n}(\theta, \varphi)$. Moreover, their time evolution in a classical magnetic field exactly matches the classical evolution [5]. Finally, there exists a representation of the density operator of an arbitrary quantum state in the form

$$
\hat{\rho}=\frac{2 j+1}{4 \pi} \int_{0}^{\pi} \sin \theta d \theta \int_{0}^{2 \pi} d \varphi P(\theta, \varphi)|\theta, \varphi\rangle\langle\theta, \varphi| .
$$

Similar to Eq. (1), this is formally equivalent to a decomposition of a quantum state into a statistical mixture of (classical) coherent states. Therefore, all quantum states, for which the weight function $P(\theta, \varphi)$ is non-negative, are classical angular momentum states. Conversely, any quantum state for which such a non-negative function $P(\theta, \varphi)$ does not exist, will be referred as being nonclassical [6, 7].

\section{B. The Holstein-Primakoff-approximation}

Let us consider a system with a large angular momentum, i.e. $j \gg 1$, which is mainly aligned in the $z$-direction. In this case, the operator $\hat{J}_{z}$ can be approximated by its expectation value $\left\langle\hat{J}_{z}\right\rangle \approx j$, being a real number as in classical physics. Only the remaining angular momentum components $\hat{J}_{x}, \hat{J}_{y}$ are treated fully quantum-mechanically [17]. Then, the ladder operators satisfy the approximated commutator relation

$$
\left[\hat{J}_{+}, \hat{J}_{-}\right] \approx 2 j
$$

The re-normalized operators $\hat{j}_{ \pm}=\frac{1}{\sqrt{2 j}} \hat{J}_{ \pm}$obey the same algebra as the bosonic operators. We may introduce rescaled angular momentum components via $\hat{j}_{ \pm}=\frac{1}{2}\left(\hat{j}_{x} \pm\right.$ $\left.\mathrm{i} \hat{j}_{y}\right)$, which are defined such that the uncertainty of the ground state, $\left.\left.\langle j ; j|\left(\Delta \hat{j}_{x}\right)^{2}\right)|j ; j\rangle=\langle j ; j|\left(\Delta \hat{j}_{y}\right)^{2}\right)|j ; j\rangle=$ 1. The weight function $P(\theta, \varphi)$ corresponds to the Glauber-Sudarshan $P$ function of the quantum state, with the complex argument $\alpha=\sqrt{j / 2} \theta e^{i \varphi}$. The properties of $P(\alpha)$ form the basis for defining nonclassicality of the harmonic oscillator. Consequently, we can reformulate established methods for nonclassicality for angular momentum systems.

\section{Atomic nonclassicality quasiprobabilities}

Although the $P$ function uniquely defines the nonclassicality of a quantum system, its reconstruction is in general impossible for the harmonic oscillator, since it can be highly singular. For getting definite answers about nonclassical effects, one may introduce a filtered atomic $P$ function, in close analogy to that of the harmonic oscillator [18. For atomic systems, one multiplies the characteristic function $\Phi(\xi)$ of $P(\theta, \varphi)$,

$$
\Phi(\xi) \equiv\left\langle e^{\xi \hat{j}_{-}-\xi^{*} \hat{j}_{+}}\right\rangle e^{|\xi|^{2} / 2},
$$

with a suitable filter function $\Omega_{w}(\xi)$ (parameterized by $w)$,

$$
\Phi_{\Omega}(\xi) \equiv \Phi(\xi) \Omega_{w}(\xi)
$$

The atomic nonclassicality quasiprobability is then given by

$$
P_{\Omega}\left(j_{x}, j_{y}\right) \equiv \frac{1}{\pi^{2}} \int_{\mathbb{C}} d^{2} \xi e^{\mathrm{i}\left(j_{y} \xi_{r}-j_{x} \xi_{i}\right)} \Phi_{\Omega}(\xi),
$$

with $\xi=\xi_{r}+\mathrm{i} \xi_{i}$. The filter function $\Omega_{w}(\xi)$ has to satisfy certain properties: First, it has to guarantee that the nonclassicality quasiprobability of any state is always regular. Second, it only should show negativities if the quantum state is nonclassical. For the harmonic oscillator we have shown [18] that for any nonclassical state one finds a nonclassicality quasiprobability demonstrating negativities by simply varying a real parameter $w$ that scales the filter width.

\section{Atomic quadrature quasiprobability}

In the following we will introduce the AQQP to efficiently characterize nonclassicality of atomic spin systems with a minimum of measured data. Quadrature distributions are just probability densities, so that a regularization procedure is superfluous. The situation changes if one analyzes the quadrature statistics beyond the ground-state noise level [19], to visualize usually hidden nonclassical effects, also beyond the negativities of the Wigner function [20]. Subsequent filtering yields the marginals of $P_{\Omega}\left(j_{x}, j_{y}\right)$. 
Introducing atomic quadrature operators $\hat{j}_{\varphi}$,

$$
\hat{j}_{\varphi} \equiv \hat{j}_{+} e^{\mathrm{i} \varphi}+\hat{j}_{-} e^{-\mathrm{i} \varphi},
$$

we define the AQQP via

$$
p_{\Omega}\left(j_{\varphi}\right) \equiv \frac{1}{2 \pi} \int_{-\infty}^{\infty} d \lambda \Phi\left(\lambda e^{\mathrm{i} \varphi}\right) \Omega_{w}(\lambda) e^{\mathrm{i} \lambda j_{\varphi}},
$$

which is just a marginal of $P_{\Omega}\left(j_{x}, j_{y}\right)$ for a fixed angle $\varphi$. Clearly, if such a marginal distribution shows negativities, then $P_{\Omega}\left(j_{x}, j_{y}\right)$ has negativities as well, and the state is nonclassical. In general, the inverse conclusion is not true.

The AQQP distribution can be sampled from a set of $N$ measured angular momentum values $\left\{\tilde{j}_{k}\right\}_{k=1}^{N}$ directly by using a similar approach to 21]. Hence an estimator for the AQQP is expressed as an empirical mean of a suitable pattern function $f_{\Omega}\left(\tilde{j} ; j_{\varphi}, w\right)$,

$$
p_{\Omega}\left(j_{\varphi}\right)=\frac{1}{N} \sum_{k=1}^{N} f_{\Omega}\left(\tilde{j}_{k} ; j_{\varphi}, w\right) .
$$

The required pattern function is given by

$$
f_{\Omega}\left(\tilde{j} ; j_{\varphi}, w\right)=\frac{1}{2 \pi} \int_{-\infty}^{\infty} e^{k^{2} / 2} e^{\mathrm{i} k\left(\tilde{j}-j_{\varphi}\right)} \Omega_{w}(k) d k .
$$

Furthermore, the uncertainty of $p_{\Omega}\left(j_{\varphi}\right)$ can be estimated as $1 / \sqrt{N}$ times the empirical standard deviation of the sampled numbers $\left\{f_{\Omega}\left(\tilde{j}_{k} ; j_{\varphi}, w\right)\right\}_{k=1}^{N}$. Therefore, the sampling method is a very simple way to estimate the quantity of interest together with its statistical error.

\section{EXPERIMENTAL PROCEDURE}

\section{A. Experimental Setup}

The setup is described in detail in 22, 23]: A cloud of $N_{a} \approx 2 \cdot 10^{5}$ cold Cesium atoms is prepared by laser cooling in a magneto-optical trap (MOT) and subsequently transferred into a far off-resonant dipole trap formed in the $40 \mu \mathrm{m}$ waist of a single $5 \mathrm{~W}, 1064 \mathrm{~nm}$ laser beam. Employing a sequence of optical pumping pulses, microwave pulses, (optical) purification pulses, a magnetic bias field of approximately 1.5 Gauss, and a resonant microwave $\pi / 2$ pulse, each atom is prepared in the state $\left|\psi_{0}\right\rangle=\left(\left|F=3, m_{F}=0\right\rangle+\left|F=4, m_{F}=0\right\rangle\right) / \sqrt{2}$.

In a simplified picture we describe each atom as a twolevel system with pseudo-spin $1 / 2$ and with basis states $\left|\frac{1}{2}, \pm \frac{1}{2}\right\rangle:=\frac{1}{\sqrt{2}}\left(|F=3\rangle \pm e^{i \omega_{34} t}|F=4\rangle\right)$, with $\hbar \omega_{34}=$ $\Delta E_{\text {hyperfine. The atomic population difference in the two }}$ hyper-fine manifolds $F=3$ and $F=4$ now can be regarded as the $x$-component of a collective $j=N_{a} / 2$ pseudospin vector. This corresponds to the sum of the individual pseudo-spins, $|j ; j\rangle=\bigotimes_{i=1}^{N_{a}}\left|\psi_{0}\right\rangle_{i}$, an ACS.
By detecting the differential phase shifts $\phi$ that the atomic cloud imprints on a bichromatic, off-resonant optical probe pulse 23 , we measure the atomic population difference $\Delta N$ between the two hyper-fine levels. In this way we perform a non-destructive quantum nondemolition (QND) measurement of $\hat{j}_{x}$, which projects the atomic state into a squeezed state around $\zeta \phi_{1}$. Here $\phi_{1}$ denotes the outcome of the QND measurement and the parameter $\zeta$ is a function of the QND measurement strength, which is directly proportional to the optical density of the ensemble and the probability of spontaneous photon emission 22. A subsequent verification measurement $\phi_{2}$, performed on the same ensemble, shows sub-projection noise quantum fluctuations of the differential phase shift with respect to the prediction $\zeta \phi_{1}$.

As it is characteristic for QND measurements the signal-to-noise ratio (and therefore the measurement strength) is limited by the quantum noise of the meter system - in our case by the shot noise $\delta n$ of the probe light:

$$
\phi_{1}=\frac{\delta n_{1}}{n_{1}}+\kappa \Delta N, \quad \phi_{2}=\frac{\delta n_{2}}{n_{2}}+\kappa \Delta N,
$$

where $\kappa$ is the light-atom coupling strength. Although the destructive second measurement uses more photons $\left(n_{2}=1.5 n_{1}\right)$ than the entangling QND measurement, it still contains a fair amount of light shot noise (see Fig 1).

\section{B. Data analysis}

To be able to convert the $N$ measurement outcomes $\phi_{12 k}:=\phi_{2 k}-\zeta \phi_{1 k}(k=1, \ldots, N)$ into quadrature samples, we calibrate our experiment as follows:

- We prepare a ACS using a varying number of trapped atoms $N_{a}$ and, for calibration purposes only, subtract measurement outcomes $\phi_{k}^{\text {ACS }}$ from successive independent MOT loading cycles $(\approx 5 \mathrm{~s}$ apart) to compensate for slow drifts of experimental parameters. By performing a scaling analysis 22 on the differential values $\bar{\phi}_{k}^{\mathrm{ACS}}=\left(\phi_{k}^{\mathrm{ACS}}-\phi_{k+1}^{\mathrm{ACS}}\right) / \sqrt{2}$ we can identify the amount of light shot noise $\operatorname{var}(\delta n / n)$ and atomic quantum projection noise $\operatorname{var}(\kappa \Delta N)$ and discriminate those against technical noise sources.

- Additionally, due to spontaneous emission processes caused by the $\phi_{1}$-measurement, performed with $n_{1}=4.1 \cdot 10^{7}$ photons, the atomic state is not pure anymore and compared to the ACS the Ramsey-fringe contrast (and thus the macroscopic spin $j$ of the ensemble) is reduced by a factor $\eta=\exp \left(-n_{1} \epsilon\right)=65.8 \%$, where $\epsilon=1.02 \cdot 10^{-8}$ is the decoherence per photon, determined experimentally as described in [23].

With these calibrations we can calculate the phase fluctuations that we would measure in the absence of any 


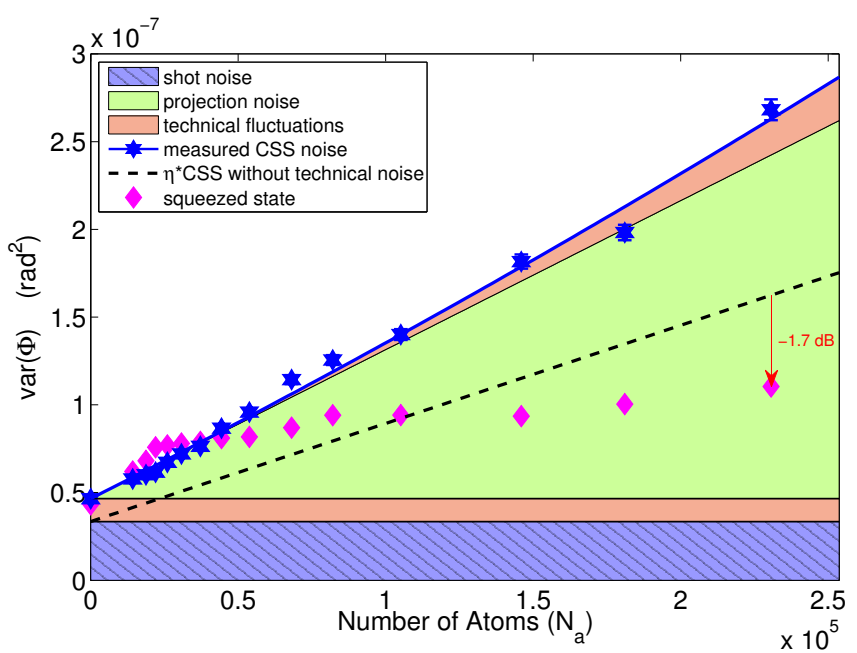

Figure 1. (Color online) Calibration procedure: Blue points: $\operatorname{var}\left(\bar{\phi}^{\mathrm{ACS}}\right)$ for different atom numbers; each data point is formed by 4841 individual measurements. The scaling of the noise as a function of the number of atoms $N_{a}$ allows us to identify noise contributions. Blue (dark shaded) area: light shot noise. Green (bright shaded) area: atomic projection noise. Red (grey shaded) areas: technical fluctuations. Black dashed line: predicted noise for a ACS with the same collective $j_{z}$ as our squeezed state. Magenta diamonds: $\operatorname{var}\left(\phi_{12}\right)$ for experiments with different $N_{a}$.

technical noise for a ACS comprised of $\eta N_{a}$ atoms, which yields the same Ramsey fringe amplitude as our squeezed state:

$$
\operatorname{var}_{\mathrm{ACS}\left(\eta N_{a}\right)} \equiv \operatorname{var}\left(\delta n_{2} / n_{2}\right)+\kappa^{2} \eta N_{a}
$$

For a varying number of atoms $0 \leq N_{a} \leq 2.9 \cdot 10^{5}$ we perform in total $N=84730$ experiments and obtain phase shift measurements $\phi_{12 k}$, now without taking the difference between subsequent MOT loading cycles.

We convert the $\phi_{12 k}$ into quadrature values by normalizing to $\operatorname{var}_{\mathrm{ACS}}\left(\eta N_{a}\right)$ :

$$
\bar{j}_{k}=\phi_{12 k} / \sqrt{\operatorname{var}_{\mathrm{ACS}\left(\eta N_{a}\right)}} .
$$

The $\bar{j}_{k}$ do not only contain the atomic signal $\tilde{j}_{k}$ but also added photon shot noise, which is Gaussian noise, uncorrelated with the atomic population difference $\Delta N$. Due to the normalization procedure the effect of such added Gaussian noise is equivalent to a detection with a reduced quantum efficiency [24] (mixing with an ACS). The effective quantum efficiency of our quadrature measurement depends on the 'atomic signal to shot noise'-ratio and is therefore a function of $N_{a}$ :

$$
\text { Efficiency }=\frac{\operatorname{var}_{\mathrm{ACS}\left(\eta N_{a}\right)}-\operatorname{var}_{\mathrm{ACS}(0)}}{\operatorname{var}_{\mathrm{ACS}}\left(\eta N_{a}\right)}
$$

In our experiment, it reaches values up to $83 \%$ for measurements taken with the highest number of atoms $N_{a}=$ $2.9 \cdot 10^{5}$. This effect only attenuates negativities of the nonclassicality quasiprobability $P_{\Omega}$ and any nonclassicality found in $\bar{j}_{k}$ is therefore sufficient to prove a nonclassical atomic state in the pseudospin operators $\hat{j}_{x}, \hat{j}_{y}$.

\section{Verification of nonclassicality}

In the following, we only consider experiments performed with an effective detection efficiency above $77 \%$, which show a quadrature variance $1.67 \mathrm{~dB}$ below the projection noise. From the $\left\{\bar{j}_{k}\right\}_{k=1}^{N=4841}$ data points, we can estimate the AQQP according to Eq. 12, by using a one-dimensional autocorrelation filter

$$
\Omega_{w}(k)=\mathcal{N}^{-1} \int \omega\left(k^{\prime}\right) \omega\left(k^{\prime}+k / w\right) d k,
$$

with $\omega(k)=e^{-k^{4}}$ and $\mathcal{N}=\int[\omega(k)]^{2} d k$. This filter preserves all information about the distribution, but only displays negativities for nonclassical states. First, we look at the significance of the negativity as a function of the filter width,

$$
\Sigma(w)=\min \left(\frac{p_{\Omega}\left(j_{\varphi}\right)}{\sigma\left(p_{\Omega}\left(j_{\varphi}\right)\right)}\right),
$$

where $\sigma\left(p_{\Omega}\left(j_{\varphi}\right)\right)$ is the standard deviation of the estimate $p_{\Omega}\left(j_{\varphi}\right)$. This quantity is nothing but the value of the negativity in terms of standard deviations of the measurement. For observing nonclassical effects, this quantity has to be negative. From our experimental data, we always observe such negative significance. Moreover, the inset in Fig. 2 shows the dependence of its absolute value on the filter width $w$. It can be seen that there is a filter width, for which the significance is optimized. In case of squeezing, this may even be arbitrarily close to $w=0$. Here, we can obtain a significance of up to 23 standard deviations.

The main part of Fig. 2 shows the AQQP of the corresponding state. Here, the filter width is chosen to be $w=1.1$, which gives a smaller significance of 10.0 standard deviations, but also leads to more pronounced effects. We observe oscillations with significant negativities. Since the autocorrelation function (Eq. (18) ) fulfills the requirements of a filter function as listed in the theory part by construction, the negativities of the AQQP clearly reflect the nonclassicality of the quantum state.

\section{CONCLUSIONS}

We introduced the atomic quadrature quasiprobability, $p_{\Omega}\left(j_{\varphi}\right)$, for atomic systems described by angular momentum states in the Holstein-Primakoff approximation. Its negativities certify negativities of the Glauber-Sudarshan $P$-function of the atomic system, and hence the nonclassicality of the state beyond both the ground-state noise level and negativities of the Wigner function. We apply our method to experimental data observed in a 


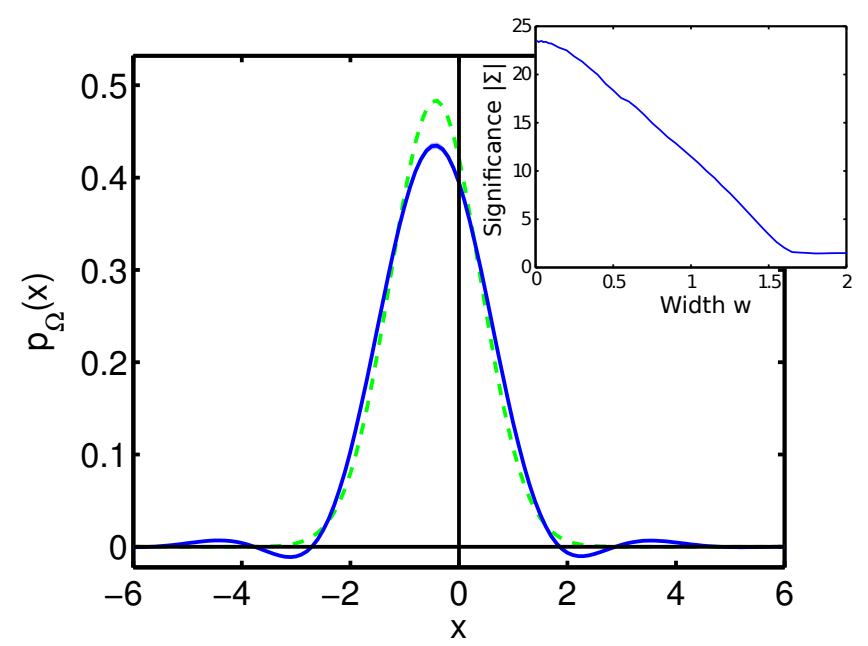

Figure 2. (Color online) AQQP distribution of a squeezed angular momentum state for a filter width $w=1.1$. The error bars are smaller than the linewidths. The dashed green curve shows the standard quadrature distribution (i.e. the marginal of the Wigner function), which is non-negative. Inset: Significance $\Sigma$ of the negativity of the AQQP versus the width parameter. squeezed atomic ensemble and demonstrate nonclassicality with a statistical significance of up to 23 standard deviations. We consider our method a valuable tool for certifying nonclassicality especially for states prepared in composed many-identical-particle systems such as cold atomic clouds, vapor cells or rare-earth doped crystals for quantum technology and -communication applications.

\section{ACKNOWLEDGMENTS}

This work was supported by the Deutsche Forschungsgemeinschaft through SFB 652, by Danmarks Grundforskningsfond, and by Q-ESSENCE.
[1] E. C. G. Sudarshan, Phys. Rev. Lett. 10, 277 (1963).

[2] R. J. Glauber, Phys. Rev. 131, 2766 (1963).

[3] U. M. Titulaer and R. J. Glauber, Phys. Rev. 140, B676 (1965).

[4] F. T. Arecchi, E. Courtens, R. Gilmore, and H. Thomas, Phys. Rev. A 6, 2211 (1972).

[5] A. Perelomov, Commun. Math. Phys. 26, 222 (1972).

[6] O. Giraud, P. Braun, and D. Braun, Phys. Rev. A 78, 042112 (2008).

[7] A. Luis, Phys. Rev. A 73, 063806 (2006).

[8] M. Kitagawa and M. Ueda, Phys. Rev. A 47, 5138 (1993).

[9] A.Kuzmich, K. Mølmer, and E. S. Polzik, Phys. Rev. Lett. 79, 4782 (1997).

[10] A. Kuzmich, N. P. Bigelow, and L. Mandel, Europhys. Lett. 42, 481 (1998).

[11] D. J. Wineland, J. J. Bollinger, W. M. Itano, F. L. Moore, and D. J. Heinzen, Phys. Rev. A 46, 6797 (1992).

[12] A. Sørensen, L. M. Duan, J. I. Cirac, and P. Zoller, Nature 409, 63 (2001).

[13] K. Hammerer, A. S. Sørensen and E. S. Polzik, Rev. Mod. Phys. 82, 1041 (2010).
[14] A. Luis and N. Korolkova, Phys. Rev. A 74, 043817 (2006).

[15] A. Luis and Á. Rivas, Phys. Rev. A 84, 042111 (2011).

[16] A. Perelomov, Generalized Coherent States and their Applications (Springer-Verlag, Berlin, 1986)

[17] T. Holstein and H. Primakoff, Phys. Rev. 58, 1098 (1940).

[18] T. Kiesel and W. Vogel, Phys. Rev. A 82, 032107 (2010).

[19] W. Vogel, Phys. Rev. Lett. 84, 1849 (2000).

[20] A. I. Lvovsky and J. H. Shapiro, Phys. Rev. A 65, 033830 (2002).

[21] T. Kiesel, W. Vogel, B. Hage, and R. Schnabel, Phys. Rev. Lett. 107, 113604 (2011).

[22] J. Appel, P. J. Windpassinger, D. Oblak, U. Busk Hoff, N. Kjærgaard, and E. S. Polzik, Proc. Natl. Acad. Sci. U.S.A. 106, 10960 (2009).

[23] A. Louchet-Chauvet, J. Appel, J. J. Renema, D. Oblak, N. Kjærgaard, and E. S. Polzik, New J. Phys. 12, 065032 (2010).

[24] J. Appel, D. Hoffman, E. Figueroa, and A. I. Lvovsky, Phys. Rev. A 75, 035802 (2007). 